\title{
The HC-Pro Protein of Potato Virus $Y$ Interacts with NtMinD of Tobacco
}

\author{
Yongsheng Jin, ${ }^{1}$ Dongyuan Ma, ${ }^{1}$ Jiangli Dong, ${ }^{1}$ Daofeng Li, ${ }^{1}$ Changwang Deng, ${ }^{1}$ Jingchen Jin, ${ }^{2}$ and \\ Tao Wang ${ }^{1}$
}

${ }^{1}$ State Key Laboratory for Agro-Biotechnology, China Agricultural University, Beijing, 100094, P.R China; ${ }^{2}$ Henan Agricultural University, Zhengzhou, 450002, P.R China

Submitted 6 June 2007. Accepted 11 July 2007.

\begin{abstract}
Potato virus $Y$ (PVY) infections often lead to altered numbers of host plant chloroplasts, as well as changes in morphology and inhibited photosynthesis. The multifunctional protein helper component-proteinase, HC-Pro, has been identified in PVY-infected leaf chloroplasts. We used yeast two-hybrid and bimolecular fluorescence complementation assays to demonstrate that HC-Pro can interact with the chloroplast division-related factor NtMinD in yeast and tobacco cells, respectively. In addition, we confirmed that residues 271 to 314 in NtMinD are necessary for its interaction with PVY HC-Pro in a yeast two-hybrid analysis using four NtMinD deletion mutants. These residues are necessary for the dimerization of NtMinD, which plays a vital role in chloroplast division. Thus, PVY HC-Pro may affect NtMinD activity by inhibiting the formation of NtMinD homodimers, and this may interfere with chloroplast division and contribute to changes in the numbers of chloroplast per cell observed in PVY-infected plants.
\end{abstract}

Additional keywords: BiFC, plastid division, Potyvirus.

Depending on the combination of virus and host, as well as the environmental conditions, Potyvirus-infected plants exhibit various symptoms, including mosaic patterns, vein banding, stunting, chlorosis, leaf distortion, and necrotic lesions (De Bokx and Huttinga 1981). The most common symptom of such plants is chlorosis, which may result from disruptions in chloroplast structure and function caused by interactions with viral genes or gene products (Pompe-Novak et al. 2001; Rahoutei et al. 2000). Interactions between viruses or specific viral gene products and host chloroplasts have been observed for a variety of virus-host combinations (Abbink et al. 2002; Culver et al. 1991; Lehto et al. 2003; Van Loon 1987).

Potato virus $Y$ (PVY) is the type member of the Potyvirus genus in the family Potyviridae (Shukla et al. 1994). PVY is a flexuous virion with a single-stranded, positive-sense RNA genome nearly $10 \mathrm{~kb}$ in length. The single open reading frame in the PVY genome encodes a polyprotein that is proc-

Dongyuan Ma and Yongsheng Jin contributed equally to this work.

Corresponding author: T. Wang; Telephone: +86 10 62733969; Fax: +8610-6273969; E-mail: wangt@cau.edu.cn

Nucleotide sequence data for $N t M i n D$ is available under accession number 911359 .

* The $\boldsymbol{e}$-Xtra logo stands for "electronic extra" and indicates that Figures 2,4 , and 5 appear in color online. essed into 10 functional proteins by three virally encoded proteases: P1, HC-Pro, and Nia (Carrington and Freed 1990).

One of the three proteinases, HC-Pro is a multifunctional protein (Maia et al. 1996). In addition to its self-cleavage from the polyprotein precursor (Carrington and Herndon 1992), it is involved in a number of processes, including aphid transmission (Govier et al. 1977), cell-to-cell (Rojas et al. 1997) and long-distance movement (Saenz et al. 2002), virulence and symptom development (Atreya et al. 1992; Redondo et al. 2001), synergy with coinfecting viruses (Pruss et al. 1997), and gene silencing suppression (Llave et al. 2000). HC-Pro interacts with itself (Urcuqui-Inchima et al. 1999) and with such virally encoded proteins as capsid protein (CP) (RoudetTaver et al. 2002), P1 (Merits et al. 1999), and VPg (Yambao et al. 2003). HC-Pro also interacts with various host proteins (Guo et al. 2003), including a calmodulin-like protein involved in gene silencing (Anandalakshmi et al. 2000). In PVYinfected plants, HC-Pro and CP have been detected in the leaf chloroplasts (Gunasinghe and Berger 1991).

Chloroplasts are essential organelles in which photosynthesis and many other fundamental intermediary metabolic reactions take place (Maple and Møller 2007). Chloroplast division is mediated by the coordinated action of FtsZ, MinD, MinE, and other factors (Maple et al. 2005). Arabidopsis MinD (AtMinD), a member of the ParA ATPase family, is a $\mathrm{Ca}^{2+}$-dependent ATPase (Aldridge and Møller 2005) that interacts with MinE and with itself. AtMinD hydrolyzes ATP to ensure precise Z-ring placement and symmetric plastid division (Aldridge and Møller 2005). AtMinD1 overexpression in tobacco produces abnormally large chloroplasts that are present in fewer numbers compared with those in wild-type tobacco plants (Dinkins et al. 2001). Similarly, transgenic Arabidopsis plants with reduced AtMinD expression exhibit variability in chloroplast size and number and asymmetric chloroplast division (Colletti et al. 2000). The $\alpha$ helix 11 at the $\mathrm{C}$-terminal end is vital to the dimerization of AtMinD, and the importance of this dimerization has been determined through the analysis of a mutant of AtMinD $(A R C 11)$. ARC11 loses the ability to dimerize and contribute to frequent asymmetric chloroplast division events, resulting from a point mutation in the C-terminal helix of AtMinD (A296G) (Fujiwara et al. 2004).

Using HC-Pro as bait, we screened a tobacco cDNA library using a yeast two-hybrid assay. We identified a gene involved in chloroplast division that interacts with HC-Pro, which we designated NtMinD. We then confirmed the interaction between $\mathrm{NtMinD}$ and HC-Pro in living plant cells using a bimolecular fluorescence complementation (BiFC) assay ( $\mathrm{Hu}$ and Kerppola 2003; $\mathrm{Hu}$ et al. 2002). In addition, we determined the domain 


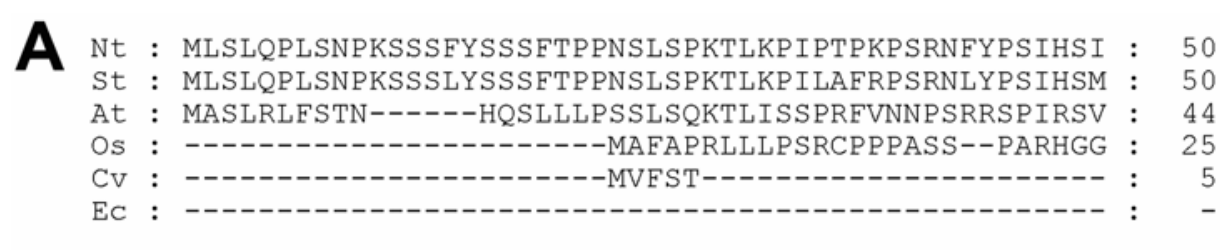

Nt : LQYNRKPQLAGETPRVVVITSGKGGVGKTTTTANIGLSLARLGFSVVAID : 100 St : LQYNRKPQLAGDTPRVLVITSGKGGVGKTTTTANIGLSLARLGFSVVAID : 100 At : LQFNRKPELAGETPRIVVITSGKGGVGKTTTTANVGLSLARYGFSVVAID : 94 Os : ---RTAPELSGPTPRVVVVTSGKGGVGKTTTTANLAASLARLSLSAVAVD : 72 $\mathrm{CV}:---G N G D D N S K G L E R V I V I T S G K G G V G K T T T T A N L G M S I A R L G Y R V A L I D \quad: \quad 52$ EC : ---------MARI IVVTSGKGGVGKTTSSAAIATGLAQKGKKTVVID : 38

Nt : CDVGLRNLDLLLGLENRVNYTVVEVLNGDCRLDQALVRDKRWSNFELLCI : 150 St : CDVGLRNLDLLLGLENRVNYTVVEVLNGDCRLDQALVRDKRWSNFELLCI : 150 At : ADLGLRNLDLLLGLENRVNYTCVEVINGDCRLDQALVRDKRWSNEELLCI : 144 Os : ADAGLRNLDLLLGLENRVHLTAADVLAGDCRLDQALVRHRALHDLQLLCL : 122 $\mathrm{CV}:$ ADIGLRNLDLLLGLENRVLYTAMDIVEGQCRLDQALIRDKRWKNLALLAI : 102 EC : FDIGLRNLDLIMGCERRVVYDFVNVIQGDATLNQALIKDKRTENLYILPA : 88

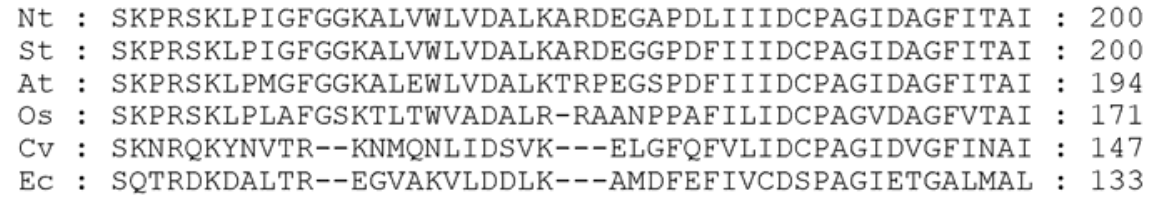

Nt : TPANEAVLVTTPDITSLRDADRVTGLLECDGIRD-------IKMMVNRV : 242 St : TPANEAVLVTTPDITSLRDADRVTGLLECDGIRD--------IKMIVNRV : 242 At : TPANEAVLVTTPDITALRDADRVTGLLECDGIRD-------IKMIVNRV : 236 Os : APAEEAVLVTTPDITALRDADRVAGLLECDGIKD--------IKIIVNRV : 213 $\mathrm{CV}:$ ASAQEAVIVTTPEITAIRDADRVAGLLEANGIYN--------VKLLVNRV : 189 EC : YFADEAIITTNPEVSSVRDSDRILGILASKSRRAENGEEPIKEHLLLTRY : 183

Nt : RTDMIKGEDMMSVLDVQEMLGLPLLGVIPEDSEVIRSTNRGYPLVLNKPP : 292 St : RTDMIKGEDMMSVLDVQEMLGLPLLGVIPEDSEVIRSTNRGYPLVLNKPP : 292 At : RTDMIKGEDMMSVLDVQEMLGLSLLGVIPEDSEVIRSTNRGFPLVLNKPP : 286 Os : RPDLVKGEDMMSALDVQEMLGLPLLGVVPEDAEVIRSTNRGVPLVLNDPP : 263 $\mathrm{CV}:$ RPDMIQKNDMMSVRDVQEMLGIPLLGAIPEDTSVIISTNKGEPLVLNKKL : 239 EC : NPGRVSRGDMLSMEDVLEILRIKLVGVIPEDQSVLRASNQGEPVILDIN- : 232
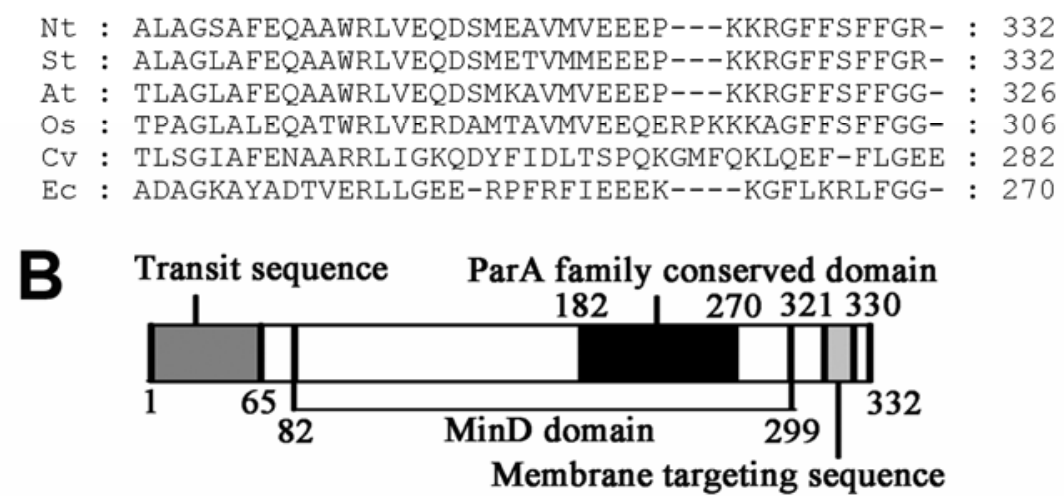

Fig. 1. Multiple sequence alignment and domains of tobacco MinD. A, Sequence alignment of MinD proteins from Nicotiana tabacum (Nt; database accession number 911359), Solanum tuberosum (St; AAZ23778), Arabidopsis (At; BAA90261), Oryza sativa (Os; NP_001056561), Chlorella vulgaris (Cv; NP_045875), and Escherichia coli (Ec; j03153). B, Schematic overview of domains of NtMinD. 
of NtMinD necessary for its interaction with HC-Pro. Our findings suggest that the interaction between HC-Pro and NtMinD may affect the function of NtMinD in chloroplast division.

\section{RESULTS}

Library screening using PVY HC-Pro as bait.

HC-Pro, which is vital for PVY infection, is also an RNAi suppressor; thus, it is expected to interact with various host factors. Accordingly, a yeast two-hybrid system was used to identify HC-Pro-interacting molecules from a tobacco cDNA library. Twenty positive clones were identified from among roughly $2 \times 10^{6}$ clones. One positive clone, which encoded a polypeptide containing 196 amino acids with a ParA ATPase domain, shared a high degree of identity with AtMinD.

The full-length cDNA of this gene was cloned by reversetranscriptase polymerase chain reaction (PCR). Based on a sequence alignment with MinD from Arabidopsis, Solanum tuberosum, Oryza sativa, Chlorella vulgaris, and Escherichia coli, we presumed that this gene encoded the tobacco MinD protein (Fig. 1A). The sequence of this putative chloroplast division-related gene, named NtMinD, has been submitted to GenBank (accession number 911359). Primary sequence of NtMinD

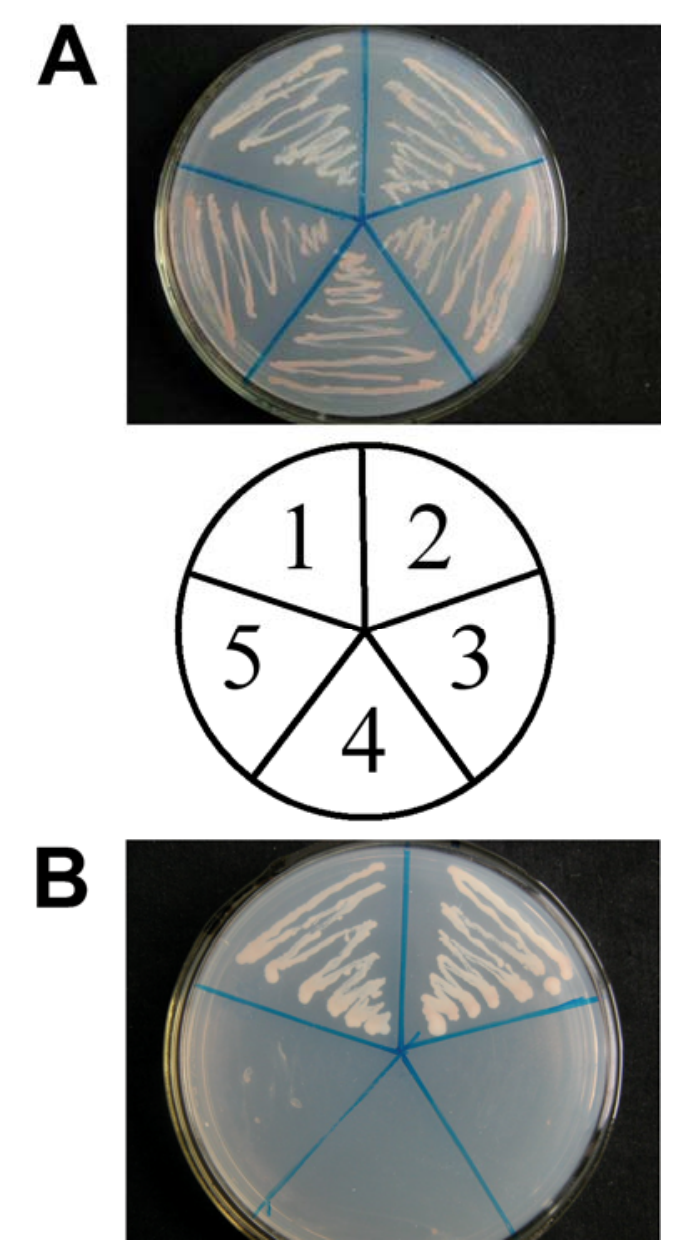

Fig. 2. Interaction of Potato virus $Y$ HC-Pro and NtMinD in Saccharomyces cerevisiae AH109 cells. A, Transformed S. cerevisiae AH109 cells grown on synthetic defined (SD)/-Leu/-Trp and $\mathbf{B}$, transformed $S$. cerevisiae AH109 cells grown on SD/-Ade/-His/-Leu/-Trp. Segment 1, pGBKT753/pGADT7-RecT (positive control); segment 2, pGBKT7-HCPro/pGADT7-NtMinD; segment 3, pGBKT7/pGADT7-NtMinD (negative control); segment 4, pGBKT7-HC-Pro/pGADT7 (negative control); and segment 5, pGBKT7/pGADT7 (negative control). contains 332 amino acids residues, which may form 12 helices predicted by the PSIPRED secondary structure prediction program. Sequence blast analysis of NtMinD in the National Center for Biotechnology Information showed that residues 1 to 65 is a transit sequence, residues 82 to 299 is a MinD domain, and residues 182 to 270 is a ParA family conserved domain (Fig. 1B). Residues 321 to 330 is a membrane-associated motif according to the membrane localization analysis ( $\mathrm{Hu}$ and Lutkenhaus 2003; Szeto et al. 2002) (Fig. 1B).

\section{HC-Pro interacts with full-length NtMinD in yeast.}

To test whether HC-Pro can interact with full-length NtMinD, the full-length coding sequence of HC-Pro was inserted in frame into the GAL4 DNA binding domain of pGBKT7, and the full-length coding sequence of NtMinD was inserted into the GAL4 activation domain of pGADT7. The resulting two plasmids, pGBKT7-HC-Pro and pGADT7-NtMinD, were cotransformed into AH109 cells, whereas pGBKT7/ pGADT7, pGBKT7/pGADT7-NtMinD, and pGBKT7-HC-Pro/ pGADT7 were cotransformed into AH109 as negative controls.

All of the transformants were able to grow on synthetic defined (SD) medium lacking His and Trp (SD/-His/-Trp), but only the pGBKT7-HC-Pro/pGADT7-NtMinD cotransformed yeast and the positive control could grow on SD medium lacking Ade, His, Leu, and Trp (SD/-Ade/-His/-Leu/-Trp) (Fig. 2). These data indicate that HC-Pro can interact with NtMinD in yeast cells.

\section{Identification of the PVY HC-Pro/NtMinD interaction in living plant cells using BiFC.}

To verify our two-hybrid results, we used BiFC to test the interaction between HC-Pro and NtMinD in living plant cells. We constructed two transient vectors expressing HC-Pro or $\mathrm{NtMinD}$ as a fusion with the $\mathrm{N}$-terminal 155 amino acids or Cterminal 86 amino acids of yellow fluorescent protein (YFP), respectively, driven by the Cauliflower mosaic virus (CaMV) $35 \mathrm{~S}$ promoter. Onion (Allium cepa) epidermal cells were bombarded with a mixture of the two constructs. Two constitutive vectors also were constructed that expressed HC-Pro or $\mathrm{NtMinD}$ as a fusion with the N-terminal 155 amino acids or Cterminal 86 amino acids of YFP, respectively, driven by the CaMV $35 \mathrm{~S}$ promoter. The two constitutive vectors were first transformed into Agrobacterium tumefaciens EHA105, then coinfiltrated into Nicotiana benthamiana. YFP fluorescence in the onion and tobacco epidermal cells was detected using laser confocal scanning microscopy.

HC-Pro interacted with NtMinD in living plant cells (Fig. 3). YFP fluorescence was detected in the onion epidermal cells $16 \mathrm{~h}$ after bombardment (Fig. 3A); no YFP fluorescence was de-

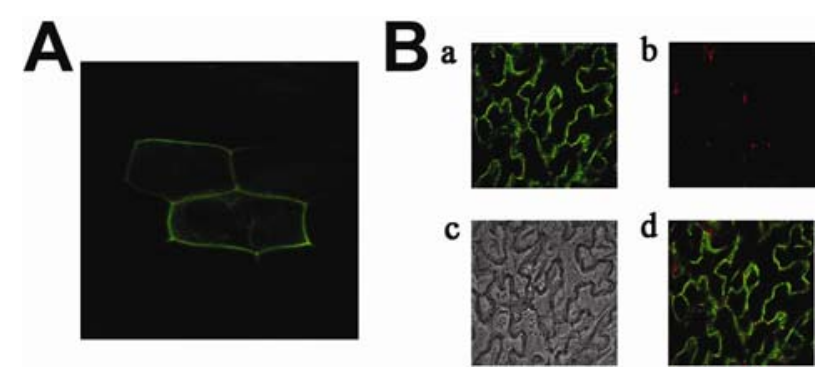

Fig. 3. Interaction of Potato virus $Y$ HC-Pro and NtMinD in living plant cells. A, Fluorescence of reconstructed YFP complexes (green) in onion (Allium cepa) epidermal cells. B, Subcellular localization of reconstructed yellow fluorescent protein (YFP) complexes determined in leaf epidermis of Nicotiana benthamiana. Section a, YFP fluorescence (green); section b, chlorophyll autofluorescence (red); section c, bright field; and section d, YFP/chlorophyll autofluorescence overlay. 
tected in the negative controls (i.e., cobombardment with pUCSPYCE-NtMinD/pUC-SPYNE, pUC-SPYCE/pUC-SPYNE-HCPro, and pUC-SPYCE/pUC-SPYNE) (data not shown). YFP fluorescence also was detected in tobacco epidermal cells that had been coinfiltrated with Agrobacterium harboring the constitutive expression vectors 35S-SPYNE-HC-Pro and 35SSPYCE-NtMinD (Fig. 3B). These results confirm that HC-Pro interacts with NtMinD in living plant cells.

\section{Identification of the NtMinD domain necessary for its interaction with PVY HC-Pro.}

To identify the domain of NtMinD that mediates its interaction with HC-Pro and to investigate the effect of HC-Pro on NtMinD function, we constructed four NtMinD deletion mutants: NtMinD1 (residues 166 to 332), which contained ParA family conserved domain and membrane targeting sequence; NtMinD2 (residues 1 to 270), which contained transit sequence and ParA family conserved domain; NtMinD3 (residues 1 to 296), which is 26 residues longer than NtMinD2; and NtMinD4 (residues 1 to 314), which contained a transit sequence, ParA family conserved domain, and MinD domain but not membrane targeting sequence (Fig. 4A). The coding sequences of the mutants were subcloned individually into pGADT7 and cotransformed with pGBKT7-HC-Pro into Saccharomyces cerevisiae AH109 cells. The domain of NtMinD necessary for its interaction with HC-Pro was determined using a yeast twohybrid assay.

All of the transformants were able to grow on SD/-His/-Trp medium, but pGADT7-NtMinD, pGADT7-NtMinD1, pGADT7-
NtMinD3, and pGADT7-NtMinD4, which were separately cotransformed with pGBKT7-HC-Pro into AH109 cells, restored the auxotrophy of the yeast cells so that the transformants were able to grow on SD/-Ade/-His/-Leu/-Trp medium (Fig. 4B). These results indicate that NtMinD, NtMinD1, NtMinD3, and NtMinD4 interacted with HC-Pro, and NtMinD2 did not interact with HC-Pro. It means that residues 1 to 270 of NtMinD are not necessary for the interaction with HC-Pro. In addition, the intensity of the HC-Pro-NtMinD4 interaction was stronger than HC-Pro-NtMinD3; therefore, it is indicated that residues 297 to 314 play an important role in the HC-Pro-NtMinD interaction. Thus, we conclude that the HC-Pro-interacting domain in NtMinD is located in residues 271 to 314 .

The role of residues 271 to 314 in NtMinD dimerization.

To test whether residues 271 to 314 are necessary for NtMinD dimerization, the deletion mutant NtMinD4 was subcloned into pGBKT7 to form pGBKT7-NtMinD4. Then, pGADT7-NtMinD, pGADT7-NtMinD1, pGADT7-NtMinD2, pGADT7-NtMinD3, and pGADT7-NtMinD4 were cotransformed with pGBKT7-NtMinD4 into yeast cells. The only deletion mutant unable to interact with NtMinD4 was NtMinD2; the other three mutants retained the ability to interact with NtMinD4 (Fig. 5). These results suggest that residues 271 to 314 but not residues 1 to 270 are necessary for NtMinD dimerization. Thus, the HC-Pro-interacting domain in NtMinD also plays an important role in the formation of $\mathrm{NtMinD}$ homodimers.

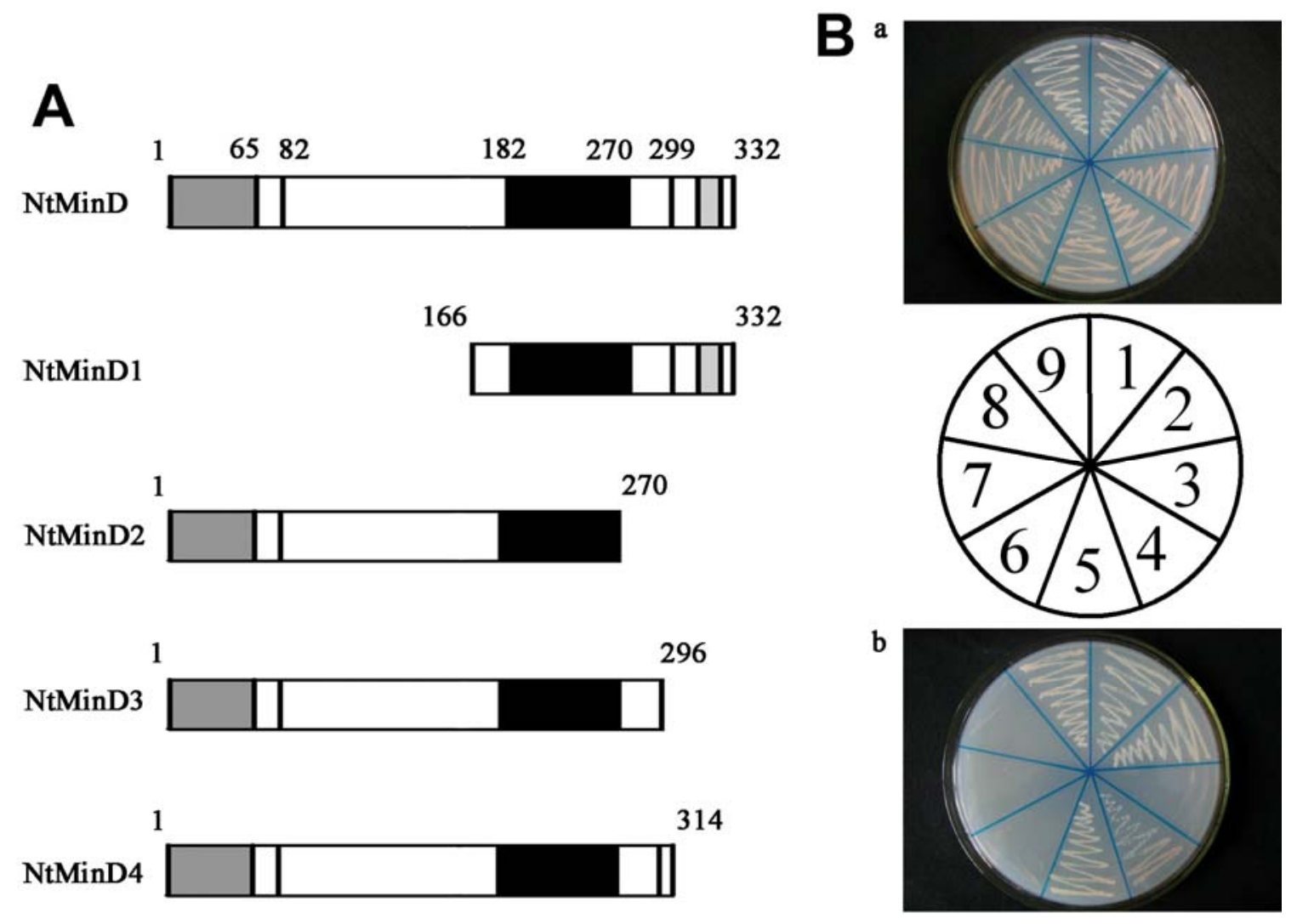

Fig. 4. Schematic overview of NtMinD mutants and the interaction of Potato virus $Y$ (PVY) HC-Pro and mutants of NtMinD in yeast cells. A, Schematic overview of NtMinD and four NtMinD mutants: NtMinD, NtMinD1, NtMinD2, NtMinD3, and NtMinD4. B, Interaction of PVY HC-Pro and mutants of NtMinD in Saccharomyces cerevisiae AH109 cells. Section a, transformed S. cerevisiae AH109 cells grown on synthetic defined (SD)/-Leu/-Trp and section b, transformed S. cerevisiae AH109 cells grown on SD/-Ade/-His/-Leu/-Trp. Segment 1, pGBKT7-HC-Pro/pGADT7-NtMinD; segment 2, pGBKT7-HCPro/pGADT7-NtMinD1; segment 3, pGBKT7-HC-Pro/pGADT7-NtMinD2; segment 4, pGBKT7-HC-Pro/pGADT7-NtMinD3; segment 5, pGBKT7-HCPro/pGADT7-NtMinD4; segment 6, pGBKT7/pGADT7-NtMinD (negative control); segment 7, pGBKT7-HC-Pro/pGADT7 (negative control); segment 8, pGBKT7/pGADT7 (negative control); and segment 9, pGBKT7-53/pGADT7-RecT (positive control). 


\section{DISCUSSION}

PVY infections can alter chloroplast numbers, morphology, and inhibit their function in affected plants (Pompe-Nonak et al. 2001); however, it is unclear how these changes occur (Nadderi and Berger 1997). Interactions between host plant proteins and viral components are presumed to play a pivotal role in the alteration. In fact, interactions between PVY or specific PVY gene products and host chloroplasts or proteins have been observed. HC-Pro and CP of PVY have been detected in the chloroplasts of infected leaves (Gunasinghe and Berger 1991). In addition, the PVY CP protein can interact with the large subunit of Rubisco in tobacco chloroplasts (Feki et al. 2005), and the CP of Turnip mosaic potyvirus interacts with a 37-kDa chloroplast protein (McClintock et al. 1998). Several host factors also have been found to interact with HC-Pro protein, such as a calmodulin-like protein involved in gene silencing (Anandalakshmi et al. 2000), a novel RING finger protein (HIP1) and HIP2 (Guo et al. 2003), but no chloroplast protein, has been found to interact with HCPro. Here, we identified that the HC-Pro protein from PVY could interact with the tobacco MinD protein which functions in chloroplast division. The HC-Pro-NtMinD interaction was detected in yeast cells and in living plant cells using a yeast two-hybrid assay and BiFC, respectively (Figs. 2 and $3)$. This is the first report on the interaction between a chloroplast protein and HC-Pro protein.

Plastid division is necessary for the maintenance and accumulation of plastids within plant cells. In plastid division, a Zring is formed by FtsZ, an ancient tubulin-like protein, to which other components of the division machinery are recruited (Maple et al. 2005). The Z-ring is localized to the plastid midpoint and correct Z-ring placement is mediated by the coordinated action of several prokaryote-derived Min proteins, including MinD and MinE (Maple et al. 2005). MinD is a $\mathrm{Ca}^{2+}$ dependent ATPase and member of the ParA ATPase family (Aldridge and Møller 2005), with a conserved membrane-association motif at its C-terminus (Szeto et al. 2002). Studies have shown that MinD plays a vital role in chloroplast division and is required for correct placement of the Z-ring. In transgenic Arabidopsis, reduced levels of AtMinD or AtMinD overexpression both can result in alterations in chloroplast size and number within individual cells (Colletti et al. 2000, Dinkins et al. 2001). The interaction of HC-Pro with NtMinD may affect the activity of NtMinD, producing asymmetric chloroplast division in PVY-infected leaves.

The domain of NtMinD that interacts with HC-Pro was identified in a yeast two-hybrid assay using deletion mutants. Our
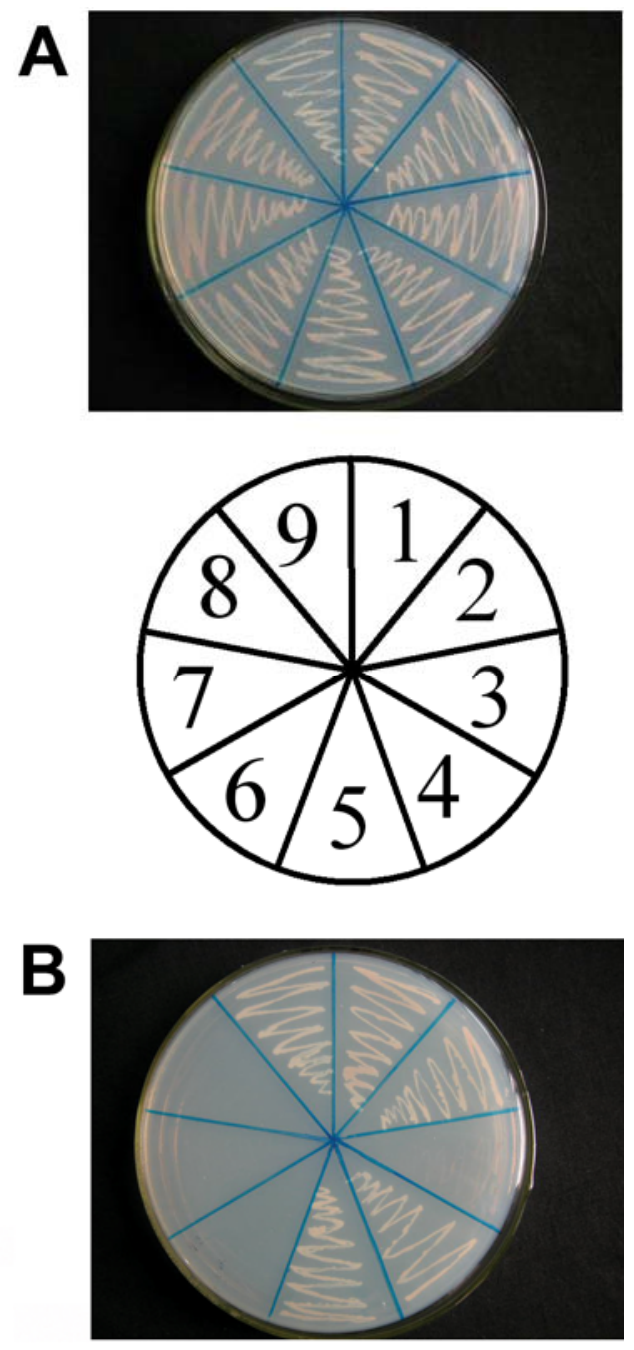

Fig. 5. Interaction of NtMinD4 and four mutants of NtMinD and NtMinD in Saccharomyces cerevisiae AH109 cells. A, Transformed S. cerevisiae AH109 cells grown on synthetic defined (SD)/-Leu/-Trp and B, transformed S. cerevisiae AH109 cells grown on SD/-Ade/-His/-Leu/-Trp. Segment 1, pGBKT7-NtMinD4/pGADT7-NtMinD; segment 2, pGBKT7-NtMinD4/ pGADT7-NtMinD1; segment 3, pGBKT7-NtMinD4/pGADT7-NtMinD2; segment 4, pGBKT7-NtMinD4/pGADT7-NtMinD3; segment 5, pGBKT7NtMinD4/pGADT7-NtMinD4; segment 6, pGBKT7/pGADT7-NtMinD (negative control); segment 7, pGBKT7-NtMinD4/pGADT7 (negative control); segment 8, pGBKT7/pGADT7 (negative control); and segment 9, pGBKT7-53/pGADT7-RecT (positive control).

Table 1. Primers used in plasmids construction

\begin{tabular}{|c|c|c|c|}
\hline Primers & Primer sequences & Restriction sites & Constructions \\
\hline HC-Pro5 & 5'-TAGGATTCTGTCGAATGCCGACAATTTT-3' & BamHI & pGBKT7-HC-Pro \\
\hline HC-Pro3 & 5'-TA $\overline{\overline{C T G C A G}}$ ACCAACTCTATAATGTTT-3' & pst $\mathrm{I}$ & $\ldots$ \\
\hline NtMinD5 & $5^{\prime}$-CACAAAAATGTTATCTCTGC- $3^{\prime}$ & ... & pMD18T-NtMinD \\
\hline NtMinD3 & 5'-ACCTTACCAAAGCCACTA - $3^{\prime}$ & $\ldots$ & $\ldots$ \\
\hline NtMinD5-1 & 5'-TAGAATTCATGTTATCTCTGCAGCCA-3' & EcoRI & pGADT7-NtMinD \\
\hline NtMinD3-1 & 5'-TACTCGAGCTAAATCACACCTAATAAAGG-3' & XhoI & $\ldots$ \\
\hline BiFC HC-Pro5 & 5'-ATTCTAGAATGTCGAATGCCGACAATTT-3' & $X b a 1$ & pUC-SPYCE-HC-Pro/ \\
\hline BiFC HC-Pro3 & 5'-ATACTAGTACCAACTCTATAATGTTTTA-3' & Spe 1 & pSPYNE-35S-HC-Pro \\
\hline BiFCNtMinD5 & 5'-ATACTAGTATGTTATCTCTGCAGCCA-3' & Spe 1 & pUC-SPYCE-NtMinD \\
\hline BiFCNtMinD3 & $5^{\prime}$-ATCTCGAGCCGCCCAAAAAACGAGAA-3' & XhoI & pSPYNE-35S-NtMinD \\
\hline NtMinD1-5 & 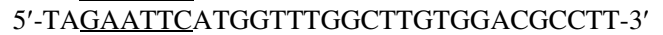 & EcoRI & pGADT7-NtMinD1 \\
\hline NtMinD1-3 & 5'-TACTCGAGCTACCGCCCAAAAAACGAGA-3' & XhoI & $\ldots$ \\
\hline NtMinD2-5 & 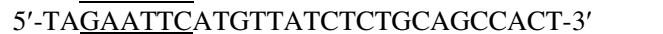 & EcoRI & $\ldots$ \\
\hline NtMinD2-3 & 5'-TA $\overline{\overline{C T C G A G}}$ CTAAATCACACCTAATAAAGG-3' & XhoI & pGADT7-NtMinD2 \\
\hline NtMinD3-3 & 5'-ATCTCGAGTTAACCTGCCAAAGCTGG-3' & XhoI & pGADT7-NtMinD3 \\
\hline NtMinD4-3 & 5'-ATCTCGAGTTAAGCCTCCATACTATCCTG-3' & XhoI & pGADT7-NtMinD4 \\
\hline NtMinD4-3-1 & 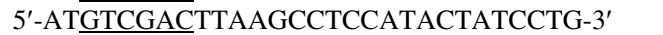 & SalI & pGBKT7-NtMinD4 \\
\hline
\end{tabular}


results indicate that residues 271 to 314 is required for its interaction with HC-Pro, rather than its transit sequence (residues 1 to 65), its ParA ATPase domain (residues 182 to 270), or its conserved C-terminal membrane-association motif (residues 321 to 330) (Fig. 4). That is to say, the function of these conserved domains may be either unaffected or indirectly affected. In addition, we found that residues 271 to 314 were necessary for the dimerization of NtMinD (Fig. 5). The importance of AtMinD dimerization in chloroplast division has been shown through the analysis of ARC11 (Fujiwara et al. 2004), and analysis of E. coli MinD mutations also showed that MinD dimerization is sufficient for MinD to bind the membrane and recruit its partners (Zhou et al. 2005). This dimerization is conserved between Arabidopsis and E. coli (Maple and Møller 2007). Therefore, we conclude that the interaction between NtMinD and HC-Pro may inhibit the formation of NtMinD homodimers, thereby affecting the normal activity of NtMinD. This may inhibit plastid division leading to the decreased chloroplast numbers and changes in the chloroplasts size observed by Pompe-Novak and associates in cells of PVY ${ }^{\mathrm{NTN}}$-infected leaves (Colletti et al. 2000; Dinkins et al. 2001; Pompe-Nonak et al. 2001).

\section{MATERIALS AND METHODS}

\section{Plant materials and growth conditions.}

$N$. tabacum L. cv. Xanthi NN was used to construct the tobacco cDNA library, whereas $N$. benthamiana was used in the BiFC assay. Surface-sterilized seed were sown on MurashigeSkoog medium to germination. The sterile tobacco plants were grown under standard greenhouse conditions.

\section{Library screen using a yeast two-hybrid assay.}

The cDNA in the N. tabacum L. cv. Xanthi library, which were expressed as fusions to the GAL4 activation domain, were constructed and cloned into pGADT7-Rec. The fulllength coding sequence of PVY HC-Pro, which was expressed as a fusion to the GAL4 DNA binding domain, was PCR amplified using PVY N strain cDNA and the primers HC-Pro5 and HC-Pro3 (Table 1). The product was cloned into pGBKT7 via the BamHI/PstI sites to produce pGBKT7-HC-Pro. We subsequently used a yeast two-hybrid system to find HC-Prointeracting proteins according to the BD Matchmaker Library Construction and Screening Kits User Manual (BD Biosciences, Franklin Lakes, NJ, U.S.A.).

\section{Cloning the full-length coding sequence of $\mathrm{NtMinD}$ from N. tabacum L. cv. Xanthi NN.}

The full-length coding sequence of NtMinD was amplified by PCR from cDNA of $N$. tabacum L. cv. Xanthi using the primers NtMinD5 and NtMinD3 (Table 1). Primer NtMinD5 was designed according to the MinD sequence from Solanum tuberosum (DQ118109), whereas primer NtMinD3 was designed according to the sequence that we obtained from our library screen. The cDNA of NtMinD was cloned into pMD18$\mathrm{T}$ (Takara) and verified by sequencing.

The full-length coding sequence of NtMinD was amplified by PCR using the primers NtMinD5-1 and NtMinD3-1 (Table 1 ), and then cloned into pGADT7 via the EcoRI/XhoI sites to form pGADT7-NtMinD. Two plasmids, pGADT7-NtMinD and pGBKT7-HC-Pro, were cotransformed into Saccharomyces cerevisiae AH109 cells as described in the BD Matchmaker Library Construction and Screening Kits User Manual. At the same time, the two plasmids supplied with the kits, pGBKT7-53 and pGADT7-RecT, were cotransformed as positive controls, whereas pGBKT7 and pGADT7, pGBKT7-HCPro and pGADT7, and pGBKT7 and pGADT7-NtMinD were cotransformed as negative controls.

\section{BiFC assay.}

The vectors (pUC-SPYNE, pUC-SPYCE, pSPYNE-35S, and pSPYCE-35S) used in the BiFC assay were kind gifts of Klaus Harter and Jörg Kudla. For BiFC analysis, the fulllength coding sequence of HC-Pro was PCR amplified using the primers BiFCHC-Pro5 and BiFCHC-Pro3 (Table 1) and cloned into pUC-SPYNE or pSPYNE-35S as a fusion with the

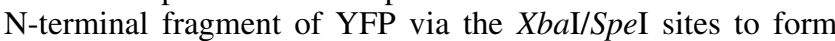
pUC-SPYNE-HC-Pro and pSPYNE-35S-HC-Pro. The fulllength coding sequence of NtMinD was PCR amplified using the primers BiFCNtMinD5 and BiFCNtMinD3 (Table 1) and then cloned into pUC-SPYCE or pSPYCE-35S, respectively, as a fusion with the C-terminal fragment of YFP via the SpeI/XhoI sites to form pUC-SPYCE-NtMinD and pSPYCE35S-NtMinD (Walter et al. 2004). The plasmids pUC-SPYNEHC-Pro and pUC-SPYCE-NtMinD were cobombarded into onion (Allium cepa) epidermal cells following the method of $\mathrm{Xu}$ and associates (2006), and YFP fluorescence was analyzed 12 to $18 \mathrm{~h}$ later (Xu et al. 2006). The relevant controls were prepared at the same time. For infiltration of $N$. benthamiana, Agrobacterium tumefaciens EHA105 was infiltrated into the abaxial air space of 2- to 4-week-old plants, and the epidermal layers of the leaves were assayed for fluorescence 2 days later (Wydro et al. 2006).

Fluorescence analysis was performed using a Nikon ECLIPSE TE2000-E inverted fluorescence microscope equipped with a Nikon D-ECLIPSE C1 spectral confocal laser scanning system. YFP fluorescence was examined at $514 \mathrm{~nm}$ (excitation) using an argon laser with an emission band of 515 to $530 \mathrm{~nm}$ and $650 \mathrm{~nm}$ (chlorophyll autofluorescence).

\section{Construction of NtMinD deletion mutants.}

NtMinD1 (residues 166 to 332), NtMinD2 (residues 1 to 270), NtMinD3 (residues 1 to 296), and NtMinD4 (residues 1 to 314) were the four mutants designed for NtMinD. The primers used in amplifying the coding sequences of these mutants were NtMinD1-5 and NtMinD1-3, NtMinD2-5 and NtMinD2-3, NtMinD2-5 and NtMinD3-3, and NtMinD2-5 and NtMinD4-3, respectively (Table 1). All of the coding sequences were subcloned into pGADT7 and individually cotransformed with pGBKT7-HC-Pro into AH109 cells to determine which domains of NtMinD are necessary for its interaction with PVY HC-Pro. In addition, the coding sequence of NtMinD4 (residues 1 to 314) was subcloned into pGBKT7 via the EcoRI/SalI sites to form pGBKT7NtMinD4.

\section{ACKNOWLEDGMENTS}

We thank K. Harter (Botanisches Institut, Universität zu Köln, Germany) and J. Kudla (Institut für Botanik und Botanischer Garten, Molekulare Entwichlungsbiologie der Pflanzen, Universität Müneter, Schlossplatz 4, 48149 Münster, Germany) for the vectors used in the BiFC assay, and H. Zhou and L. Liang for help with the confocal microscope.

\section{LITERATURE CITED}

Abbink, T. E., Peart, J. R., Mos, T. N., Baulcombe, D. C., Bol, J. F., and Linthorst, H. J. 2002. Silencing of a gene encoding a protein component of the oxygen-evolving complex of photosystem II enhances virus replication in plants. Virology 295 (2):307-319.

Aldridge, C., and Møller, S. G. 2005. The plastid division protein AtMinD1 is a $\mathrm{Ca}^{2+}$-ATPase stimulated by AtMinE1. J. Biol. Chem. 280:3167331678.

Anandalakshmi, R., Marathe, R., Ge, X., Herr, J. M., Jr., Mau, C., Mallory, A., Pruss, G., Bowman, L., and Vance, V. B. 2000. A calmodulin-related protein that suppresses posttranscriptional gene silencing in plants. Science 290:142-144.

Atreya, C. D., Atreya, P. L., Thornbury, D. W., and Pirone, T. P. 1992. Site- 
directed mutations in the potyvirus HC-Pro gene affect helper component activity, virus accumulation, and symptom expression in infected tobacco plants. Virology 191:106-111.

Carrington, J. C., and Freed, D. D. 1990. Cap-independent enhancement of translation by a plant potyvirus $5^{\prime}$ nontranslated region. J. Virol. 64:1590-1597.

Carrington, J. C., and Herndon, K. L. 1992. Characterization of the potyviral HC-Pro autoproteolytic cleavage site. Virology 187:308-315.

Colletti, K. S., Tattersall, E. A., Pyke, K. A., Froelich, J. E., Stokes, K. D., and Osteryoung, K. W. 2000. A homologue of the bacterial cell division site-determining factor MinD mediates placement of the chloroplast division apparatus. Curr. Biol. 10:507-516.

Culver, J. N., Lindbeck, A. G. C., and Dawson, W. O. 1991. Virus-host interactions: Induction of chlorotic and necrotic responses in plants by tobamoviruses. Annu. Rev. Phytopathol. 29:193-217.

De Bokx, J. A., and Huttinga, H. 1981. Page 242 in: CMI/AAB Descriptions of Plant Viruses. A. Gibbs, B. D. Harrison, and A. F. Murant, eds. Commonwealth Mycological Institute, Kew, U.K.

Dinkins, R., Reddy, M. S., Leng, M., and Collins, G. B. 2001. Overexpression of the Arabidopsis thaliana MinD1 gene alters chloroplast size and number in transgenic tobacco plants. Planta 214:180-188.

Feki, S., Loukili, M. J., Triki-Marrakchi, R., Karimova, G., Old, I., Ounouna, H., Nato, A., Nato, F., Guesdon, J.-L., Lafaye, P., and Ben Ammar Elgaaied, A. 2005. Interaction between tobacco ribulose-1-5-biphosphate carboxylase/oxygenase large subunit (RubisCO-LSU) and the PVY coat protein (PVY-CP). Eur. J. Plant. Pathol. 112:221-234.

Fujiwara, M. T., Nakamura, A., Itoh, R., Shimada, Y., Yoshida, S., and Møller, S. G. 2004. Chloroplast division site placement requires dimerization of the ARC11/AtMinD1 protein in Arabidopsis. J. Cell. Sci. 117:2399-2410.

Govier, D. A., Kassanis, B., and Pirone, T. P. 1977. Partial purification and characterization of the potato virus $\mathrm{Y}$ helper component. Virology 78:306-314.

Gunasinghe, U. B., and Berger, P. H. 1991. Association of Potato virus $Y$ gene products with chloroplasts in tobacco. Mol. Plant-Microbe Interact. 4:452-457.

Guo, D., Spetz, C., Saarma, M., and Valkonen, J. P. 2003. Two potato proteins, including a novel RING finger protein (HIP1), interact with the potyviral multifunctional protein HC-pro. Mol. Plant-Microbe Interact. 16:405-410.

Hu, C. D., and Kerppola, T. K. 2003. Simultaneous visualization of multiple protein interactions in living cells using multicolor fluorescence complementation analysis. Nat. Biotechnol. 21:539-545.

Hu, Z. L., and Lutkenhaus, J. 2003. A conserved sequence at the C-terminus of MinD is required for binding to the membrane and targeting MinC to the septum. Mol. Microbiol. 47:345-355,

Hu, C. D., Chinenov, Y., and Kerppola, T. K. 2002. Visualization of interactions among bZIP and Rel family proteins in living cells using bimolecular fluorescence complementation. Mol. Cell. 9:789-798.

Lehto, K., Tikkanen, M., Hiriart, J. B., Paakkarinen, V., and Aro, E. M. 2003. Depletion of the photosystem II core complex in mature tobacco leaves infected by the flavum strain of Tobacco mosaic virus. Mol. Plant-Microbe Interact. 16:1135-1144.

Llave, C., Kasschau, K. D., and Carrington, J. C. 2000. Virus-encoded suppressor of posttranscriptional gene silencing targets a maintenance step in the silencing pathway. Proc. Natl. Acad. Sci. U.S.A. 97:13401-13406.

Maia, I. G., Haenni, A., and Bernardi, F. 1996. Potyviral HC-Pro: A multifunctional protein. J. Gen. Virol. 77 (Pt 7):1335-1341.

Maple, J., and Møller, S.G. 2007. Plastid division: Evolution, mechanism and complexity. Ann. Bot. 99:565-579.

Maple, J., Aldridge, C., and Møller, S. G. 2005. Plastid division is mediated by combinatorial assembly of plastid division proteins. Plant $\mathrm{J}$. 43:811-823.

McClintock, K., Lamarre, A., Parsons, V., Laliberte, J. F., and Fortin, M. G. 1998. Identification of a $37 \mathrm{kDa}$ plant protein that interacts with the turnip mosaic potyvirus capsid protein using anti-idiotypic-antibodies. Plant. Mol. Biol. 37:197-204.

Merits, A., Guo, D., Jarvekulg, L., and Saarma, M. 1999. Biochemical and genetic evidence for interactions between potato A potyvirus-encoded proteins $\mathrm{P} 1$ and $\mathrm{P} 3$ and proteins of the putative replication complex. Virology 263:15-22.

Naderi, M., and Berger, P. H. 1997. Effects of chloroplast targeted potato virus $\mathrm{Y}$ coat protein on transgenic plants. Physiol. Mol. Plant. Pathol. 50:67-83.

Pompe-Novak, M., Wrischer, M., and Ravnikar, M. 2001. Ultrastructure of chloroplasts in leaves of potato plants infected by potato virus $\mathrm{Y}^{\mathrm{NTN}}$. Phyton-ann. Rei. Bot. A 41:215-226.

Pruss, G., Ge, X., Shi, X. M., Carrington, J. C., and Bowman Vance, V. 1997. Plant viral synergism: The potyviral genome encodes a broadrange pathogenicity enhancer that transactivates replication of heterologous viruses. Plant Cell 9:859-868.

Rahoutei, J., Garcia-Luque, I., and Barón, M. 2000. Inhibition of photosynthesis by viral infection: Effect on PSII structure and function. Physiol. Plant. 110:286-292.

Redondo, E., Krause-Sakate, R., Yang, S. J., Lot, H., Le Gall, O., and Candresse, T. 2001. Lettuce mosaic virus pathogenicity determinants in susceptible and tolerant lettuce cultivars map to different regions of the viral genome. Mol. Plant-Microbe Interact. 14:804-810.

Rojas, M. R., Zerbini, F. M., Allison, R. F., Gilbertson, R. L., and Lucas, W. J. 1997. Capsid protein and helper component-proteinase function as potyvirus cell-to-cell movement proteins. Virology 237:283-295.

Roudet-Tavert, G., German-Retana, S., Delaunay, T., Delecolle, B. Candresse, T., and Le Gall, O. 2002. Interaction between potyvirus helper component-proteinase and capsid protein in infected plants. J. Gen. Virol. 83:1765-1770.

Saenz, P., Salvador, B., Simon-Mateo, C., Kasschau, K. D., Carrington, J. C., and Garcia, J. A. 2002. Host-specific involvement of the HC protein in the long-distance movement of potyviruses. J. Virol. 76:1922-1931.

Shukla, D. D., Ward, C. W., and Brunt, A. A. 1994. The Potyiridae. CAB International, Wallingford, U.K.

Szeto, T. H., Rowland, S. L., Rothfield, L. I., and King, G. F. 2002. Membrane localization of MinD is mediated by a C-terminal motif that is conserved across eubacteria, archaea, and choroplasts. Proc. Natl. Acad. Sci. U.S.A. 99:15693-15698.

Urcuqui-Inchima, S., Maia, I. G., Drugeon, G., Haenni, A. L., and Bernardi, F. 1999. Effect of mutations within the cys-rich region of potyvirus helper component-proteinase on self-interaction. J. Gen. Virol. 80:2809-2812.

Van Loon, L. C. 1987. Disease induction by plant viruses. Adv. Virus. Res. 33:205-255

Voinnet, O., Rivas, S., Mestre, P., and Baulcombe, D. 2003. An enhanced transient expression system in plants based on suppression of gene silencing by the p19 protein of tomato bushy stunt virus. Plant J. 33:949956.

Walter, M., Chaban, C., Schutze, K., Batistic, O., Weckermann, K., Nake, C., Blazevic, D., Grefen, C., Schumacher, K., Oecking, C., Harter, K., and Kudla, J. 2004. Visualization of protein interactions in living plant cells using bimolecular fluorescence complementation. Plant J. 40:428438.

Wydro, M., Kozubek, E., and Lehmann, P. 2006. Optimization of transient Agrobacterium-mediated gene expression system in leaves of Nicotiana benthamiana. Acta Biochim. Pol. 53:289-298.

Xu, X., Chen, C., Fan, B., and Chen, Z. 2006. Physical and functional interactions between pathogen-induced Arabidopsis WRKY18, WRKY40, and WRKY60 transcription factors. Plant Cell 18:1310-1326.

Yambao, M. L., Masuta, C., Nakahara, K., and Uyeda, I. 2003. The central and C-terminal domains of VPg of Clover yellow vein virus are important for VPg-HCPro and VPg-VPg interactions. J. Gen. Virol. 84:28612869.

Zhou, H., Schulze, R., Cox, S., Saez, C., Hu, Z., and Lutkenhaus, J. 2005. Analysis of MinD mutations reveals residues required for MinE stimulation of MinD ATPase and residues required for MinC interaction. J. Bacteriol. 187:629-638.

\section{AUTHOR-RECOMMENDED INTERNET RESOURCE}

The PSIPRED secondary structure prediction server: bioinf.cs.ucl.ac.uk/psipred/psiform.html 